\title{
Imaging the Cytokine Receptor CXCR4 in Atherosclerotic Plaques with the Radiotracer ${ }^{68}$ Ga-Pentixafor for PET
}

\author{
Fabien Hyafil ${ }^{1,2}$, Jaroslav Pelisek ${ }^{3}$, Iina Laitinen ${ }^{1}$, Margret Schottelius ${ }^{4}$, Miriam Mohring ${ }^{1}$, Yvonne Döring ${ }^{5}$, \\ Emiel P.C. van der Vorst ${ }^{5}$, Michael Kallmayer ${ }^{3}$, Katja Steiger ${ }^{6}$, Andreas Poschenrieder ${ }^{4}$, Johannes Notni ${ }^{4}$, \\ Johannes Fischer ${ }^{7}$, Christine Baumgartner ${ }^{7}$, Christoph Rischpler ${ }^{1-8}$, Stephan G. Nekolla ${ }^{1-8}$, Christian Weber ${ }^{5-9}$, \\ Hans-Henning Eckstein ${ }^{3}$, Hans-Jürgen Wester ${ }^{4}$, and Markus Schwaiger ${ }^{1-8}$ \\ ${ }^{I}$ Department of Nuclear Medicine, Klinikum Rechts der Isar, Munich, Germany; ${ }^{2}$ Department of Nuclear Medicine, Bichat University \\ Hospital, Assistance Publique-Hôpitaux de Paris, Inserm 1148, DHU FIRE, University Diderot, Paris, France; ${ }^{3}$ Department of \\ Vascular and Endovascular Surgery, Klinikum Rechts der Isar, Munich, Germany; ${ }^{4}$ Pharmaceutical Radiochemistry, Technische \\ Universität München, Garching, Germany; ${ }^{5}$ Institute for Cardiovascular Prevention, Ludwig-Maximilians-Universität München, \\ Munich, Germany; ${ }^{6}$ Institute of Pathology, Technische Universität München, Munich, Germany; ${ }^{7}$ Centre of Preclinical Research, \\ Klinikum Rechts der Isar, Munich, Germany; ${ }^{8}$ DZHK (Deutsches Zentrum für Herz-Kreislauf-Forschung e.V.) partner site Munich \\ Heart Alliance, Munich, Germany; and ${ }^{9}$ Cardiovascular Research Institute Maastricht (CARIM), Maastricht University, Maastricht, \\ The Netherlands
}

\begin{abstract}
${ }^{68} \mathrm{Ga}$-pentixafor is a radiotracer for PET that binds with nanomolar affinity to CXCR4. The CXCR4 receptor is expressed at the surface of inflammatory cells. The objective of the study was to analyze the ability of radiolabeled pentixafor to detect CXCR4 expression on inflammatory cells present in atherosclerotic plaques of an experimental rabbit model. Methods: Atherosclerotic plaques were induced by endothelial abrasion of the right carotid artery and abdominal aorta of 7 rabbits fed an atherogenic diet. Five noninjured rabbits fed a chow diet were used as controls. Rabbits were imaged on a PET/MR system after injection of ${ }^{68} \mathrm{Ga}$-pentixafor ( $\left.15 \mathrm{MBq} / \mathrm{kg}\right)$. Vascular signal was quantified as tissue-to-background ratio (TBR). Biodistribution and autoradiographic studies were performed $1 \mathrm{~h}$ after injection of ${ }^{125}$ I-pentixafor $(7.5 \mathrm{MBq} / \mathrm{kg})$. In addition, blocking studies were performed in 2 atherosclerotic rabbits with preinjection of the CXCR4 inhibitor AMD3100. Tracer uptake was quantified on arterial cryosections using autoradiography and compared with CXCR4 and RAM-11 (macrophage) expression on adjacent histologic sections. Results: One hour after injection of ${ }^{68} \mathrm{Ga}$-pentixafor, strong signals were detected in vivo with $\mathrm{PET} / \mathrm{MR}$ imaging in atherosclerotic plaques of the abdominal aorta and right carotid artery as compared with normal control arteries (mean TBR $=1.95 \pm 0.51$ vs. $1.22 \pm 0.25$ and mean TBR $=1.24 \pm 0.38$ vs. $0.96 \pm 0.37$, respectively; $P<0.05$ for both). Blocking studies with preinjection of a CXCR4 inhibitor reduced ${ }^{125} /$-pentixafor uptake in atherosclerotic plaques by approximately $40 \%$. ${ }^{125}$-pentixafor uptake in the vessel wall on autoradiographies was located in macrophage-rich regions of atherosclerotic plaques and correlated with the intensity of CXCR4 expression on corresponding cryosections $\left(r^{2}=0.61 ; P<\right.$ $0.05)$. Conclusion: ${ }^{68} \mathrm{Ga}$-pentixafor allows for the noninvasive detection of CXCR4 expression in the vessel wall with PET and emerges as a potential alternative to ${ }^{18} \mathrm{~F}-\mathrm{FDG}$ for the assessment of macrophage infiltration in atherosclerotic plaques.
\end{abstract}

Received Jun. 20, 2016; revision accepted Sep. 29, 2016.

For correspondence or reprints contact: Fabien Hyafil, Department of Nuclear Medicine, Klinikum Rechts der Isar, Ismaningerstrasse 22, 81675 Munich, Germany.

E-mail: fabien.hyafil@gmail.com

Published online Oct. 27, 2016.

COPYRIGHT (C 2017 by the Society of Nuclear Medicine and Molecular Imaging.
Key Words: PET; atherosclerosis; radiotracer; inflammation; CXCR4; macrophages

J Nucl Med 2017; 58:499-506

DOI: 10.2967/jnumed.116.179663

T the past $10 \mathrm{y},{ }^{18} \mathrm{~F}$-FDG PET has proved a reliable noninvasive imaging approach to assess the degree of inflammation present in atherosclerotic plaques both in animal models of atherosclerosis and in patients $(1,2)$. Patients with the highest ${ }^{18} \mathrm{~F}-\mathrm{FDG}$ uptake in plaques present a higher rate of cardiovascular events and worse prognosis than patients with low vascular ${ }^{18} \mathrm{~F}-\mathrm{FDG}$ uptake (3). ${ }^{18}$ F-FDG PET detects inflammation with high sensitivity but has important limitations in the specific setting of cardiovascular imaging: physiologic uptake in the heart and in the brain, background signal of blood, interaction with blood glucose, need for a long fasting period, or dedicated diet before imaging (4). Consequently, radiotracers that more specifically target inflammatory cells might greatly facilitate the noninvasive detection of inflammatory activity in the vessel wall with PET.

${ }^{68} \mathrm{Ga}$-pentixafor is the first PET agent that exhibits high affinity and selectivity for C-X-C chemokine receptor type 4 (CXCR4) (5). The CXCR4 is a constitutive cytokine receptor involved in several biologic processes including the entry of HIV, the development of metastasis, and the evolution of different autoimmune diseases such as rheumatoid arthritis, systemic lupus erythematosus, and multiple sclerosis (6). CXCR4 expression has been identified in more than 30 different types of cancers, and its activation is a key trigger for enhanced tumor growth and progression, tumor invasiveness, and metastasis. ${ }^{68} \mathrm{Ga}$-pentixafor was therefore first evaluated in several tumors expressing CXCR4 in animal models and demonstrated both high and specific uptake in CXCR4-positive tumors and metastases (7). Proof-of-concept studies have recently been performed with ${ }^{68} \mathrm{Ga}$-pentixafor in patients with lymphoma (8) and multiple myeloma (9), both tumor entities expressing high levels of CXCR4, and demonstrated intense uptake in tumor 
cells, excellent pharmacokinetics, and favorable dosimetry in humans (10), with a low residual signal in the blood. Furthermore, CXCR4 plays a pivotal role in the trafficking of inflammatory cells by mediating the homing of progenitor cells in the bone marrow and regulating their mobilization into peripheral tissues on injury (6). In fact, the small molecular antagonist AMD3100 (plerixafor) blocks the CXCR4 receptor and allows for mobilization of hematopoietic stem and progenitor cells in the bone marrow for autografting on myeloablative treatment (11). Because of the overexpression of CXCR4 at the surface of cells involved in the inflammatory process, we hypothesized in this study that ${ }^{68} \mathrm{Ga}$-pentixafor might find a role in the detection of inflammatory cells with PET. Moreover, CXCR4 expression is strongly increased under hypoxic conditions through the stimulation of the hypoxia-inducible factor (12). ${ }^{68} \mathrm{Ga}$-pentixafor therefore represents a promising candidate for the detection of high-risk atherosclerotic plaques, which contain intense inflammatory reaction and hypoxic conditions.

The aim of this study was to test whether ${ }^{68} \mathrm{Ga}$-pentixafor specifically binds to CXCR4-expressing tissues and can be detected in atherosclerotic plaques induced in the abdominal aorta and right carotid artery of rabbits. In addition, we assessed the expression of CXCR4 in carotid plaques in a small group of patients with ${ }^{68} \mathrm{Ga}-$ pentixafor PET imaging and on histologic sections to evaluate whether the results observed in a rabbit model with ${ }^{68} \mathrm{Ga}$-pentixafor may translate in the evaluation of human atherosclerotic plaques.

\section{MATERIALS AND METHODS}

\section{Radiolabeling of Pentixafor}

Radiosynthesis of ${ }^{68} \mathrm{Ga}$-Pentixafor. ${ }^{68} \mathrm{Ga}$ was obtained by elution of $\mathrm{a}^{68} \mathrm{Ge} /{ }^{68} \mathrm{Ga}$ generator with $\mathrm{SnO} 2$ matrix (iTHEMBA LABS) with $1 \mathrm{M}$ $\mathrm{HCl}(5.5 \mathrm{~mL})$ and immobilized on a strong cationic exchanger cartridge (SCX-Chromafix, size M; Macherey-Nagel).

For animal studies, ${ }^{68} \mathrm{Ga}$-pentixafor was prepared on a Gallelut + system in analogy to a previously published ${ }^{68} \mathrm{Ga}$-labeling procedure (SCINTOMICS GmbH) (13). Briefly, ${ }^{68} \mathrm{Ga}$ generator eluate fractions (1.25 mL, 600-800 MBq, buffered to $\mathrm{pH} 3.3$ with $900 \mu \mathrm{L}$ of a solution of $14.4 \mathrm{~g} \mathrm{~N}$-(2-hydroxyethyl)piperazine- $N^{\prime}$-(2-ethanesulfonic acid) in $12 \mathrm{~mL}$ of water) were reacted with $3.5 \mathrm{nmol}$ of pentixafor (SCINTOMICS $\mathrm{GmbH}$ ) for $5 \mathrm{~min}$. The radiochemical purity was always greater than 99\% as confirmed by thin-layer chromatography and high-performance liquid chromatography (HPLC). Addition of $1 \mathrm{~mL}$ of phosphatebuffered saline and concentration in vacuo to $1 \mathrm{~mL}$ of total volume yielded solvent-free formulations with specific activities ranging from 100 to $150 \mathrm{MBq} / \mathrm{nmol}$.

For patient studies, the synthesis of ${ }^{68} \mathrm{Ga}$-pentixafor was performed in a fully automated, good manufacturing practices-compliant procedure using a GRP module (SCINTOMICS GmbH) equipped with a disposable single-use cassette kit (ABX) including a standardized labeling sequence (14). Upon being washed with water, ${ }^{68} \mathrm{Ga} 3+$ was eluted into the reaction vessel (containing $20 \mu \mathrm{g}$ of pentixafor in $3 \mathrm{~mL}$ of $1.5 \mathrm{M} N$-(2-hydroxyethyl)piperazine- $N^{\prime}$-(2-ethanesulfonic acid)) using $1.7 \mathrm{~mL}$ of $5 \mathrm{M} \mathrm{NaCl}$. The reaction mixture was heated to $140^{\circ} \mathrm{C}$ for $10 \mathrm{~min}$ and, upon cooling, transferred onto a C18 light cartridge (Waters). The cartridge was washed with water to remove unreacted ${ }^{68} \mathrm{Ga}$ activity, and ${ }^{68} \mathrm{Ga}$-pentixafor was eluted using $2 \mathrm{~mL}$ of $\mathrm{EtOH} /$ water (50/50). The eluate was diluted with $12 \mathrm{~mL}$ of phosphatebuffered saline and passed through a sterile filter. The radiochemical purity of the ready-to-inject formulation $(14 \mathrm{~mL})$ was always greater than $99 \%$ as confirmed by radio-HPLC and thin-layer chromatography, and the specific activity was in the range of 30-65 $\mathrm{GBq} / \mu \mathrm{mol}$.

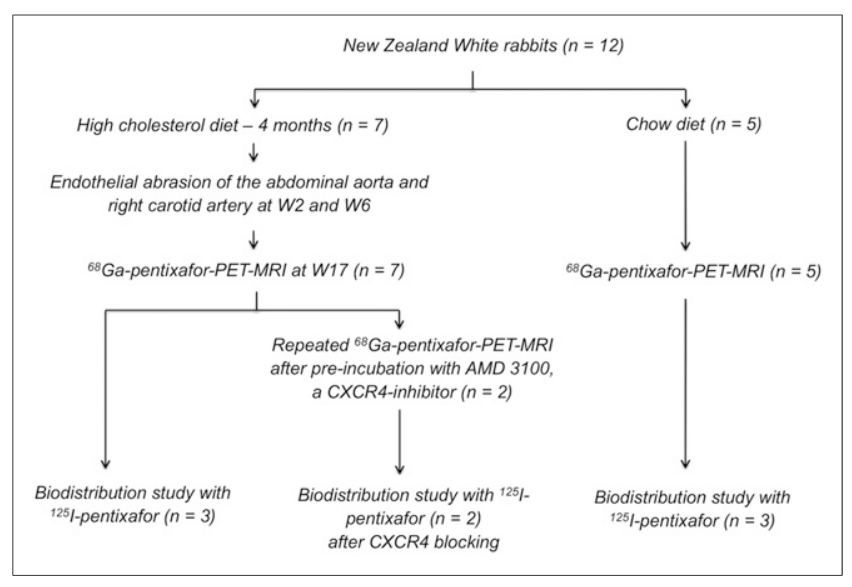

FIGURE 1. Flow chart of experimental study.

Radiosynthesis of ${ }^{125}$ I-Pentixafor. For radioiodination, 100-200 $\mu \mathrm{g}$ of pentixafor (cold gallium(III) complex) were dissolved in $0.5 \mathrm{~mL}$ of TRIS iodination buffer ( $25 \mathrm{mM}$ Tris. $\mathrm{HCl}, 0.4 \mathrm{M} \mathrm{NaCl}, \mathrm{pH} 7.5)$ and transferred to an Eppendorf reaction tube coated with $150 \mu \mathrm{g}$ of IodoGen. Upon addition of ${ }^{125} \mathrm{I}-\mathrm{Na}$ (18-20 MBq; Hartmann Analytik), the reaction vessel was briefly vortexed and the labeling reaction was allowed to proceed for $15 \mathrm{~min}$ at room temperature. The peptide solution was then removed from the insoluble oxidizing agent. Separation of ${ }^{125} \mathrm{I}$-pentixafor from unlabeled precursor was achieved using gradient reversed-phase HPLC (column: Nucleosil $100 \mathrm{C} 18$ [5 $\mu \mathrm{m}, 125 \times 4.0 \mathrm{~mm}$; CS GmbH]; gradient: $22 \%-42 \%$ ethanol [0.5\% acetic acid] in water [0.5\% acetic acid] within $20 \mathrm{~min}$; flow: $1 \mathrm{~mL} / \mathrm{min}$ ). The high-performance liquid chromatography product fraction was used as such and diluted to the required concentration using phosphate-buffered saline.

\section{Imaging of Atherosclerotic Plaques in Rabbits}

Animal Model. Atherosclerotic plaques were induced in male New Zealand White rabbits $(n=7$; mean age, 4 mo; mean weight $=3.5 \pm$ $0.2 \mathrm{~kg}$ ) by a combination of 4 mo of a high-cholesterol diet $(0.3 \%$ cholesterol-enriched diet; Altromin Spezialfutter $\mathrm{GmbH}$ ) and a double balloon injury of the aorta and the right carotid artery (2 and $6 \mathrm{wk}$ after starting the high-cholesterol diet). Endothelial abrasion was performed in the aorta with a 4-French Fogarty embolectomy catheter (Edward Lifescience $\mathrm{GmbH}$ ) introduced through the femoral artery as previously described $(2,15)$ and in the right carotid artery with a 3-French Fogarty embolectomy catheter (Edward Lifescience GmbH). All procedures were performed under general anesthesia by continuous intravenous injection of propofol and analgesia with fentanyl. Nonoperated New Zealand White rabbits, fed a normal chow diet, were used as controls $(n=5)$. Experiments were approved by the local animal care committee and were in accordance with the German Animal Welfare Act.

Study Design. In vivo imaging was performed with ${ }^{68} \mathrm{Ga}$-pentixafor PET/MR imaging in a total of 12 rabbits, including 7 rabbits that developed injury-induced atherosclerotic plaques in the abdominal aorta and right carotid artery and 5 noninjured control rabbits (Fig. 1). In 2 atherosclerotic rabbits, ${ }^{68} \mathrm{Ga}$-pentixafor PET/MR imaging was repeated after the blocking of CXCR4 obtained by the preinjection of an established CXCR4 inhibitor (AMD3100; plerixafor; SigmaAldrich) at a dose equivalent to $20 \mathrm{mmol}(\approx 2000$-fold concentration of pentixafor). Immediately after the last imaging session, animals were injected with ${ }^{125}$ I-pentixafor, and biodistribution studies were performed $1 \mathrm{~h}$ after injection in 8 rabbits (5 atherosclerotic rabbits, including 2 with blocking of CXCR4 and 3 control rabbits). 
PET/MR Imaging Protocols. Imaging was performed on a human whole-body simultaneous PET/MR (3T) scanner (Biograph mMR; Siemens). Rabbits were anesthetized with an intravenous infusion of Propofol (2\%, $\sim 1.2-1.3 \mathrm{mg} / \mathrm{kg} / \mathrm{min}$; Propofol 2\% MCT Fresenius, Fresenius Kabi Deutschland $\mathrm{GmbH}$ ) and intubated, and catheters were placed in ear veins. Rabbits positioned prone in the scanner were ventilated (Servo-I; Maquet Critical Care AB) and monitored (pulse oxymeter and electrocardiogram) during the whole imaging session. PET and MR images were acquired simultaneously in all rabbits and started $45 \mathrm{~min}$ after injection of $15 \mathrm{MBq} / \mathrm{kg}$ of ${ }^{68} \mathrm{Ga}$-pentixafor. Detailed PET and MR acquisition protocols are provided in the section "PET and MR Acquisition Protocols."

Image Analysis. Images were analyzed by an experienced nuclear medicine physician masked to the rabbit group and to the results of histology. Image registration and fusion of the time-of-flight MR acquisitions and attenuation-corrected PET images were performed with an Osirix workstation (OsiriX version 3.5.1 64-bit; OsiriX Imaging Software), an open-source DICOM viewer and image manipulator using the Horn algorithm, a manual rigid registration algorithm based on multiple points $(16,17)$. Matching of both datasets was considered as correct when rabbit contours of PET and MR images were perfectly aligned. If required (e.g., in the case of animal motion), alignment was corrected manually using the Fusion tool of the OsiriX software. For biodistribution studies, $\mathrm{SUV}_{\text {mean }}$ calculated as decay-corrected tissue radioactivity divided by body weight and injected dose was measured in circular regions of interest placed on each organ located using the MR images.

For the quantification of the vascular uptake, circular regions of interest were placed on axial adjacent PET images of the abdominal aorta and both carotid arteries (12 axial sections each) identified using the time-of-flight images. $\mathrm{SUV}_{\max }$ was recorded in each region of interest. In addition, the $\mathrm{SUV}_{\text {mean }}$ of blood was measured by placing a circular region of interest in the right atria. The tissue-tobackground ratio (TBR) was calculated as the ratio of $\mathrm{SUV}_{\max }$ and the background venous activity measured as $\mathrm{SUV}_{\text {mean }}$ of blood (18). Mean TBRs were calculated for each arterial territory as the average of TBR values throughout the entire arterial segment.

\section{PET and MR Acquisition Protocols}

MR Acquisition Protocol. MR imaging was performed using a clinical knee coil for the aorta and a bilateral 4-channel phased array coil for carotid arteries (4-channel, Machnet BV). MR acquisition first started with a localizer scan to define the bed position and was followed by a time-of-flight 3-dimensional angiographic sequence to visualize the abdominal aorta. Subsequently, a coronal 2-point Dixon 3-dimensional volumetric interpolated breath-hold T1-weighted (VIBE) MRI sequence was acquired for the generation of attenuation maps ( $\mu$-maps) and anatomic images in association with the PET acquisition. The Dixon MRI sequence was set as follows: integrated parallel acquisition technique; acceleration factor, 2; voxel size, $3.7 \times 2.3 \times 2.7 \mathrm{~mm}^{3}$ (in-plane resolution $\times$ slice thickness); acquisition time, $19 \mathrm{~s}$; repetition time, $3.6 \mathrm{~ms}$; first echo time, $1.23 \mathrm{~ms}$; second echo time, $2.46 \mathrm{~ms}$; matrix, $192 \times 192$; number of excitations, 1; field of view, $450 \mathrm{~mm}$; phase field of view, $65.6 \%$; 1 slab with 128 slices; slice thickness, $2.73 \mathrm{~mm}$; flip angle, $10^{\circ}$; and bandwidth, $965 \mathrm{~Hz} /$ pixel. The software of the MRI scanner automatically used the raw images to generate 4 different images : T1-weighted in-phase, T1-weighted out-of-phase, water-only, and fat-only. The same MR acquisition protocol was repeated for the imaging of carotid arteries.

PET Acquisition Protocol. Static emission scans were acquired during $10 \mathrm{~min}$ in a single bed position $50 \mathrm{~min}$ after the injection of ${ }^{68} \mathrm{Ga}$-pentixafor in the abdominal aorta and $60 \mathrm{~min}$ after the injection for carotid arteries. Data were corrected for randoms, dead time, scatter, and attenuation based on the $\mu$-maps generated from the Dixon images (19). Twomm-thick axial PET images were reconstructed with a $50 \times 50 \mathrm{~cm}^{2}$ field of view and a $344 \times 344$ matrix using a 3 -dimensional attenuation-weighted ordered-subsets expectation maximization iterative reconstruction algorithm with 3 iterations and 21 subsets, a zoom of 1, and no postreconstruction filter. Spatial resolution of the reconstructed PET images was previously determined to be $4.3 \mathrm{~mm}$ at $1 \mathrm{~cm}$ from the scanner's isocenter and 5.0 $\mathrm{mm}$ at $10 \mathrm{~cm}$ in the transverse direction in full width at half maximum (20).

\section{Tracer Biodistribution and Histology}

Tracer Biodistribution in Tissues. At the end of the imaging session, 8 rabbits (atherosclerotic rabbits, $n=5$; control rabbits, $n=3$ ) were injected intravenously with $7.5 \mathrm{MBq} / \mathrm{kg}$ of ${ }^{125}$ I-pentixafor into the ear vein under isoflurane anaesthesia for biodistribution studies. Because of the long half-life of ${ }^{125} \mathrm{I}$ (60 d), ${ }^{125}$ I-pentixafor allowed for more accurate biodistribution studies and autoradiographies in comparison to ${ }^{68} \mathrm{Ga}$-pentixafor (half-life, $68 \mathrm{~min}$ ). The animals were sacrificed $60 \mathrm{~min}$ after injection by intravenous injection of $150 \mathrm{mg} / \mathrm{kg}$ of pentobarbiturate PET/MR imaging system. Note high uptake of ${ }^{68} \mathrm{Ga}$-pentixafor on multiplanar reconstructions was nearly completely blocked by the preinjection of AMD3100, potent CXCR4 inhibitor. Strong accumulation of ${ }^{68} \mathrm{Ga}$-pentixafor was also found in kidneys (asterisks), reflecting renal clearance of tracer. In addition, high focal activities were detected in abdominal aorta (red arrowheads) and right carotid artery (orange arrowheads) of atherosclerotic rabbits, whereas no significant signal could be detected in noninjured left carotid artery (white arrowheads) of atherosclerotic and control rabbits as well as in abdominal aorta and right carotid artery of control rabbits. Furthermore, focal activities detected with PET in atherosclerotic plaques of abdominal aorta and right carotid artery decreased significantly when same rabbit was reimaged after blocking CXCR4 receptors.

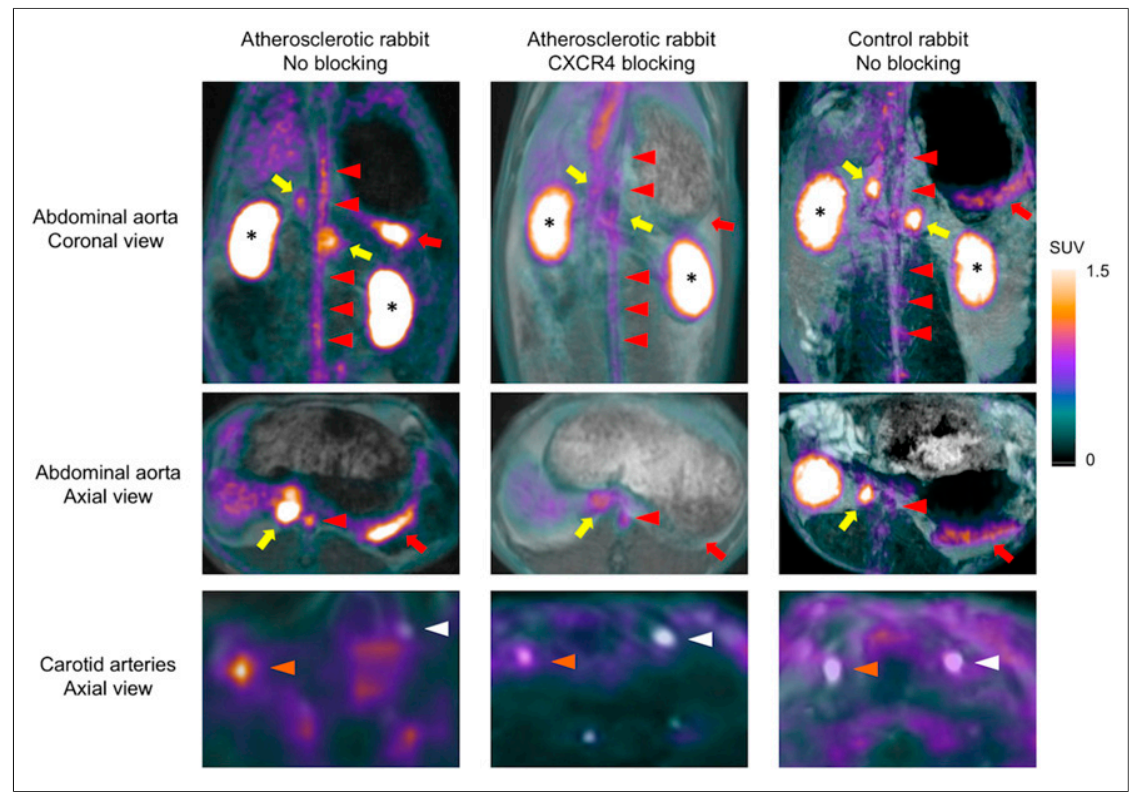




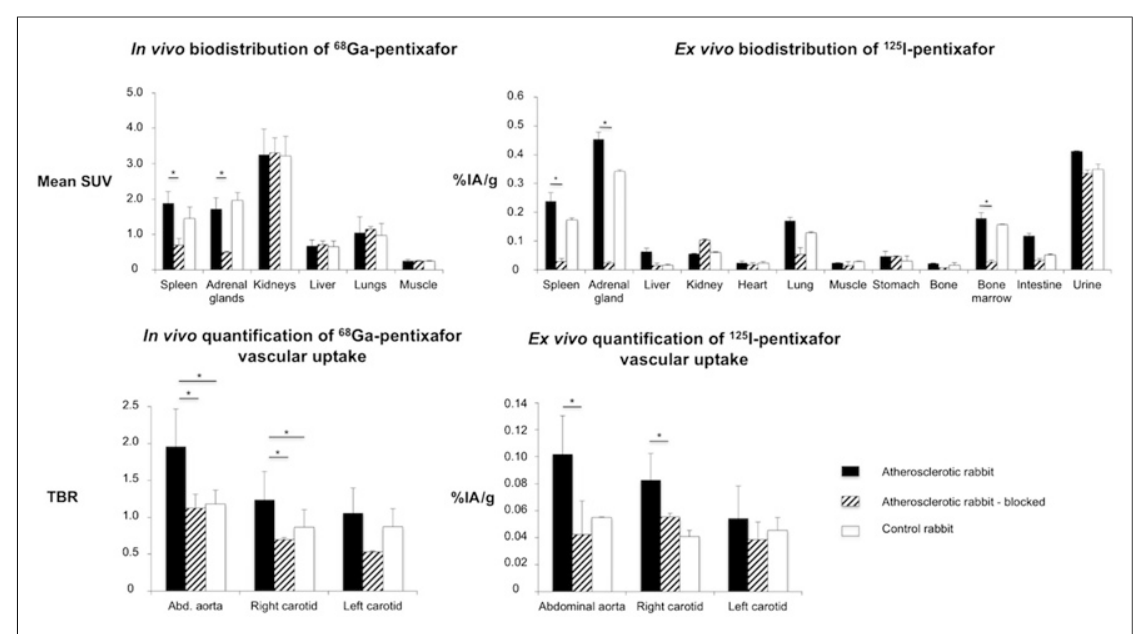

FIGURE 3. Biodistribution of radiolabeled pentixafor in rabbits. Highest activity was located in kidneys, which reflects renal-mediated clearance of radiotracer. Blocking of CXCR4 receptors significantly reduced binding of ${ }^{68} \mathrm{Ga}$-pentixafor in spleen and in adrenal glands in favor of specific uptake of radiotracer in these organs. In addition, increased uptake of ${ }^{68} \mathrm{Ga}$-pentixafor was measured in abdominal aorta and right carotid artery of atherosclerotic vs. control rabbit and was also reduced by about $40 \%$ after CXCR4 blocking. Ex vivo biodistribution studies confirmed high uptake of ${ }^{125}$-pentixafor in spleen, adrenal glands, and bone marrow, organs that express constitutively high levels of CXCR4. Activities measured in these organs were significantly lower when blocking CXCR4 receptors with preinjection of AMD3100. Activities were significantly higher in abdominal aorta and in right carotid artery, in which atherosclerotic plaques had developed, in comparison to noninjured left carotid artery of atherosclerotic rabbits and to aorta and carotid arteries of control rabbits. In addition, activities measured in atherosclerotic segments were significantly lower when blocking CXCR4 receptor. ${ }^{*} P<0.05$.

(Narcoren; Merial), and the organs of interest including the aorta and carotid arteries were dissected. The radioactivity was measured in weighted tissue samples using a $\gamma$-counter (1480 Wizard; PerkinElmer Wallac). Data are expressed in $\% \mathrm{IA} / \mathrm{g}$.

Autoradiographies were performed on $20-\mu$-thick cryosections. After an overnight exposure to image plates (Fuji TR; Fuji Photo Film Co.), the plates were scanned with an image plate reader (CR 35 BIO; Dürr Medical, Raytest Isotopenmeßgeräte $\mathrm{GmbH}$ ). The mean activity was quantified in regions of interest encompassing tissues and corrected for background signal with an image analysis program (AIDA Image Analyser; Raytest Isotopenmeßgeräte). Radiotracer uptake in sections was expressed as the mean activity corrected for background and adjusted to the mean activity measured in the vascular wall of the left carotid artery used as a reference for each plate.

Histology of Rabbit Tissues. Five- $\mu$ m-thick cryosections were fixed for $10 \mathrm{~min}$ in acetone. CXCR4 expression and macrophages were detected by immunohistochemistry using mouse monoclonal biotinylated antibodies directed against CXCR4 (clone MAB170 [R\&D Systems]; dilution, 1:500) and RAM-11 (M0633 [Dako]; dilution, 1:3,000), respectively. Primary antibodies were visualized using a peroxidase-diaminobenzidine mouse detection kit (Vectastain; Vector Laboratories). Unspecific primary immunoglobulin $\mathrm{G}$ antibodies were used as negative controls. Total CXCR4-positive area was measured digitally on each section using an automated, contrast-based, area analysis function of the Image Scope software (Aperio; Vista). The percentage of CXCR4-positive cells was calculated as the ratio between pixels with high contrast and pixels with low contrast using the automated analysis tool of the software Image Scope.

Histology of Human Carotid Plaques. Atherosclerotic plaque samples $(n=10)$ obtained from carotid endarterectomy were fixed in formalin, embedded in parrafin, and sectioned in 5- $\mu \mathrm{m}$-thick sections. CXCR4 expression and macrophages were detected by immu- nostaining with the use of the following primary antibodies: anti-CXCR4 (ab124824 [Abcam Inc.]; dilution, 1:300); anti-CD68 (clone KP1 [Dako]; dilution, 1:2,000); anti-CD31 (clone JC70A [Dako]; dilution, 1:40); anti-CD45 (clone 2B11 [Dako]; dilution, 1:100). After primary antibody incubation, visualization was performed using a peroxidase-diaminobenzidine ChemMate Detection Kit (Dako). Biotinylated antibody against CXCR4 was detected by a streptavidinhorseradish peroxidase complex (Jackson Immunoresearch Laboratories), visualized with diaminobenzidine (Sigma-Aldrich), and counterstained with Mayer hematoxylin. Unspecific primary immunoglobulin $\mathrm{G}$ antibodies were used as negative controls.

Expression of CXCR4 on Macrophage Cell Surface. Human peripheral blood mononuclear cells were isolated using densitygradient centrifugation with polymorphprep (Axis Shield). Isolated cells were plated for attachment in monocyte attachment medium, according to the manufacturer's protocol (C28051; PromoCell). Subsequent differentiation was performed using Macrophage Generation Media DXF according to the manufacturer's protocol (C28055; PromoCell). After 10 $\mathrm{d}$ of differentiation, cells were detached and replated as 200,000 cells/well (48-well plates). Cells were stimulated with statins (Fluvadin, 5 $\mu \mathrm{M}, 48 \mathrm{~h}$; Selleckchem), TNF (25 ng/mL, $24 \mathrm{~h}$; Sigma), oxLDL ( $25 \mu \mathrm{g} / \mathrm{mL}, 48 \mathrm{~h}$; KB Kalen), or a combination. Afterward, CXCR4 expression was measured using FACS analysis (CXCR4-eF450 with CD45-FITC and CD14-APC as controls, all eBioscience).

\section{Imaging of Atherosclerotic Plaques in Patients}

${ }^{68} \mathrm{Ga}$-pentixafor was synthesized and administered according to the requirements of the German Medicinal Products Act (Arzneimittelgesetz $\$ 132 b$ ) and with the approval of the responsible local regulatory authority (Regierung von Oberbayern). Before the investigation began, all patients gave written informed consent to participate. The data analysis was approved by the responsible local ethics committees. Four patients with carotid stenosis greater than $50 \%$ and 4 patients without any significant carotid stenosis $(<30 \%)$ were identified among patients who underwent ${ }^{68} \mathrm{Ga}$-pentixafor PET/MR imaging with neck acquisitions and after exclusion of patients with high ${ }^{68} \mathrm{Ga}$-pentixafor uptake in tumors adjacent to carotid arteries. Patients were imaged on average $45 \pm 4$ min after injection of $170 \pm 15 \mathrm{MBq}$ of ${ }^{68}$ Ga-pentixafor. Carotid arteries were located using the coronal 2-point Dixon 3-dimensional volumetric interpolated breath-hold T1-weighted MRI sequence used for attenuation correction. Mean TBR was quantified on 8 consecutive PET axial sections centered on each carotid bulb using the same methodology as in the animal model. In addition, the most-diseased-segment TBR was calculated for each carotid artery as the average of 3 consecutive axial sections centered on the section with the highest TBR.

\section{Statistical Analysis}

Numeric values are expressed as mean \pm SD. Statistical analysis was performed using SPSS software (SPSS Inc.). Comparisons of quantitative values measured in 2 groups were performed using a 2-tailed Student $t$ test if the number of values was greater than 20 in each group and using a Wilcoxon signed-rank test if the number of values was less than 20 in each group. For fluorescence-activated cell sorting 


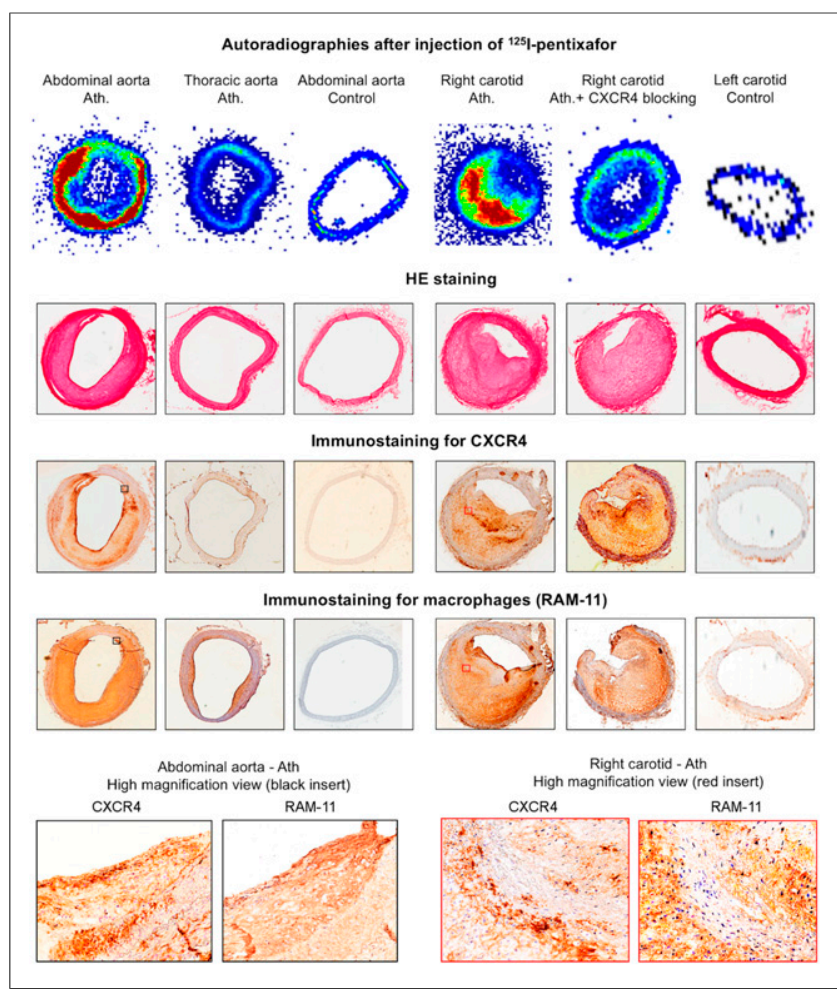

FIGURE 4. Localization of ${ }^{125}$-pentixafor accumulation in plaques. Intense activities (red color) were detected on autoradiographies of abdominal aorta and right carotid artery from atherosclerotic rabbits and were associated with high expression of CXCR4 and high density of macrophages (RAM-11 staining) in corresponding regions on immunohistochemistry of adjacent sections. In atherosclerotic rabbits preinjected with potent CXCR4 inhibitor AMD3100, low activities (blue color) were detected in atherosclerotic plaques on autoradiography, whereas intense expression of CXCR4 was present in adjacent sections on histology. In noninjured thoracic aorta and left carotid artery of rabbits fed high-fat diet and in arteries of control rabbits, only low activities (blue color) were detected on autoradiography and associated with low CXCR4 expression and rare macrophages in vascular wall by immunohistochemistry. All autoradiographic images were acquired using same color scale. Ath $=$ atherosclerotic; HE $=$ hematoxylin and eosin.

analysis, the levels of CXCR4 expression in the different groups were compared with a Kruskal-Wallis test followed by a Dunn multiple comparison test. Pentixafor uptake on autoradiography and CXCR4 expression on corresponding sections were compared using multiple regression to take into account that 3 samples were analyzed in each animal. $P$ values of less than 0.05 were considered as significant.

\section{RESULTS}

\section{Detection of CXCR4 with ${ }^{68} \mathrm{Ga}$-Pentixafor in Rabbit Model of Atherosclerotic Plaques}

In Vivo Imaging of Atherosclerotic Plaques with ${ }^{68} \mathrm{Ga}$-Pentixafor PET/MRI. Fifty minutes after intravenous injection of ${ }^{68} \mathrm{Ga}-$ pentixafor, the radiotracer accumulated in the spleen $\left(\mathrm{SUV}_{\text {mean }}=\right.$ $1.7 \pm 0.3)$ and in adrenal glands $\left(\mathrm{SUV}_{\text {mean }}=1.9 \pm 0.7\right)$, organs known to express physiologically CXCR4 receptors (Fig. 2). The highest activity was located in the kidneys $\left(\mathrm{SUV}_{\text {mean }}=3.2 \pm\right.$ $0.5)$, which reflects the renal-mediated clearance of the radiotracer. The specificity of ${ }^{68} \mathrm{Ga}$-pentixafor uptake in the spleen and in adrenal glands was confirmed by blocking CXCR4 recep- tors with a preinjection of AMD3100, which significantly reduced the signal in these organs $\left(\mathrm{SUV}_{\text {mean }}=1.7 \pm 0.3 \mathrm{vs} .0 .7 \pm 0.2\right.$ for the spleen and $\mathrm{SUV}_{\text {mean }}=1.9 \pm 0.7$ vs. $0.5 \pm 0.1$ for adrenal glands, $P<0.05$ for both; Fig. 3). In addition, focal areas of ${ }^{68} \mathrm{Ga}-$ pentixafor uptake were detected in the abdominal aortas and right carotid artery of atherosclerotic rabbits by PET imaging, whereas no significant uptake was detected in the aortas and carotid arteries of atherosclerotic rabbits as well as in control rabbits after blocking the CXCR4 receptor. ${ }^{68} \mathrm{Ga}$-pentixafor uptake in atherosclerotic plaques was quantified in vivo as the average TBR over the different arterial segments (Fig. 3). Higher TBRs were measured in the abdominal aorta and right carotid artery as compared with normal control arteries (mean TBR $=1.95 \pm 0.51$ vs. $1.22 \pm$ 0.25 and mean TBR $=1.24 \pm 0.38$ vs. $0.96 \pm 0.37 ; P<0.05$ for both). TBRs measured in the abdominal aorta and in the right carotid artery were significantly reduced $(\sim 40 \%)$ after blocking CXCR4 receptors (mean TBR $=1.95 \pm 0.51$ vs. $1.13 \pm 0.52$ and mean TBR $=1.24 \pm 0.38$ vs. $0.70 \pm 0.03 ; P<0.05$ for both).

Biodistribution of Radiolabeled Pentixafor in Rabbits. The percentage injected activity adjusted for weight (\%IA/g) was quantified $1 \mathrm{~h}$ after the injection of ${ }^{125} \mathrm{I}$-pentixafor in the different organs with and without blocking CXCR4 receptors (Fig. 3). Biodistribution studies confirmed the binding of radiolabeled pentixafor

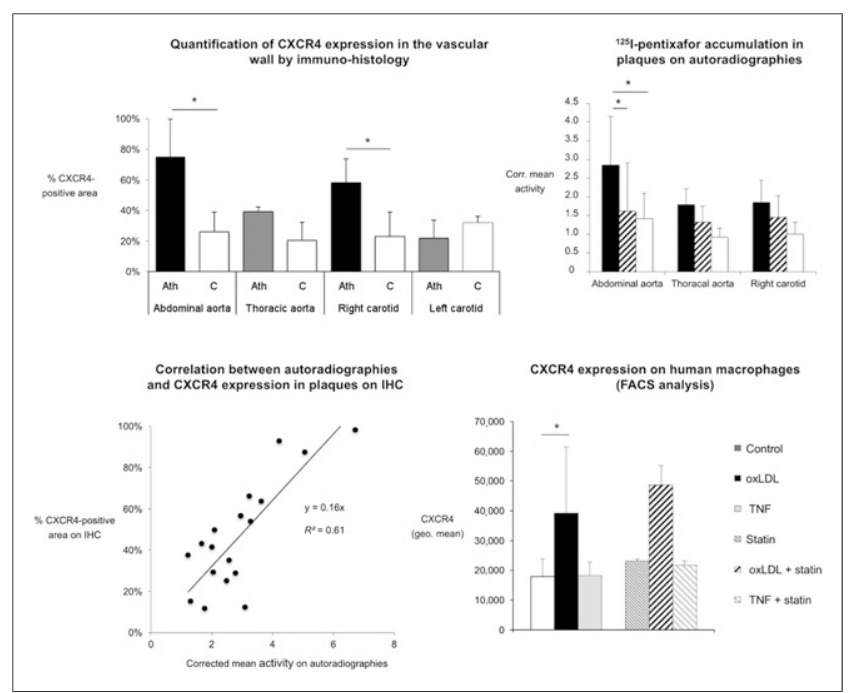

FIGURE 5. Accumulation of ${ }^{125}$-pentixafor uptake in CXCR4-expressing macrophages of atherosclerotic plaques. Quantification of activities measured in arterial wall on autoradiography confirmed higher accumulation of 125I-pentixafor in abdominal aorta and in right carotid artery in comparison to noninjured thoracic aorta and left carotid artery of atherosclerotic rabbits and to aorta and carotid arteries of control rabbits. CXCR4 expression in vascular wall was also higher in injured segments of abdominal aorta and right carotid artery of atherosclerotic rabbits in comparison to corresponding noninjured segments of control rabbits. Strong correlation was found between mean activities measured in arterial segments on autoradiography and percentage of CXCR4-positive areas quantified on adjacent histologic sections measured on immunohistology. On cell culture of macrophages, addition of oxidized low-density lipoprotein induced strong increase in expression of CXCR4 at cell surface detected with fluorescence-activated cell sorting analysis, whereas incubation with tumor necrosis factor a did not change CXCR4 expression on macrophages. Incubation with statins did not have any effect on CXCR4 expression at surface of macrophages. ${ }^{*} P<0.05$. Ath $=$ atherosclerotic; $\mathrm{C}=$ control; FACS = fluorescence-activated cell sorting; $\mathrm{IHC}=$ immunohistochemistry; oxLDL = oxidized low-density lipoprotein; TNF = tumor necrosis factor. 


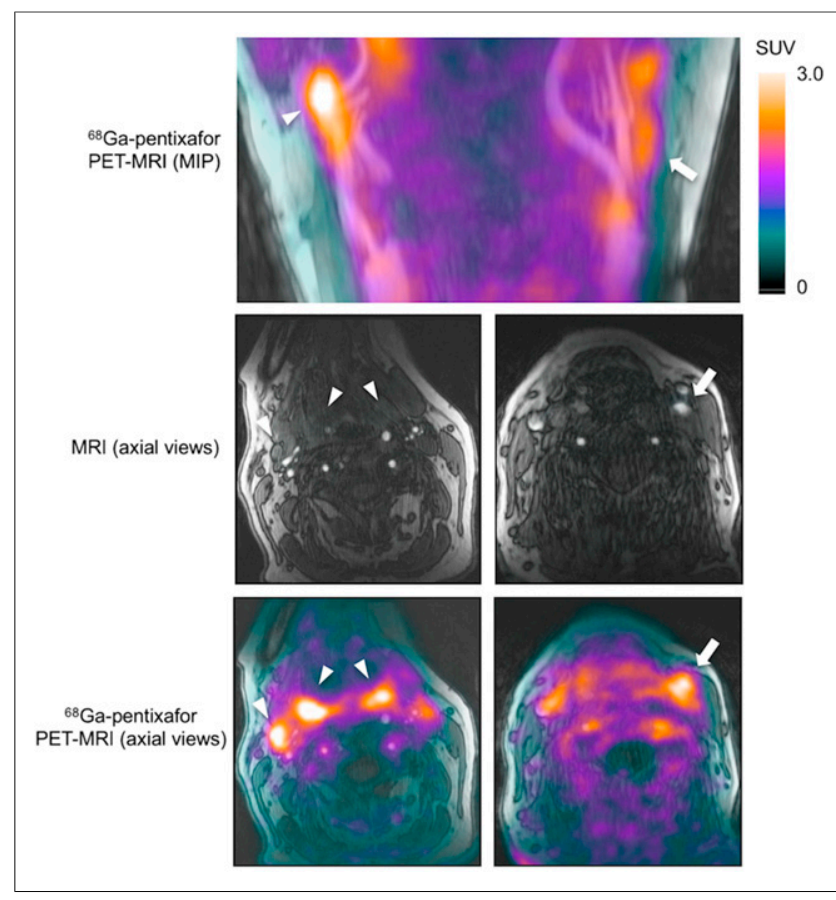

FIGURE 6. In vivo imaging of human carotid atherosclerotic plaques with ${ }^{68} \mathrm{Ga}$-pentixafor PET/MRI. Representative example of focal accumulation of ${ }^{68} \mathrm{Ga}$-pentixafor in carotid atherosclerotic plaque detected with PET/MRI. Interestingly, this patient had presented for 2 wk ischemic stroke ipsilateral to carotid plaque showing high ${ }^{68} \mathrm{Ga}$-pentixafor uptake (white arrows). Note also intense accumulation of ${ }^{68} \mathrm{Ga}$-pentixafor in perivascular lymph node and in tonsils (white arrowheads). MIP = maximum-intensity projection.

in adrenal glands, spleen, and bone marrow, which was significantly reduced in rabbits blocked with a dose of $20 \mathrm{mmol}$ of AMD3100 $(\approx 2,000$-fold molar excess over pentixafor), an established CXCR4 inhibitor, before injection of ${ }^{125} \mathrm{I}$-pentixafor $(0.33 \pm 0.14 \mathrm{vs}$. $0.02 \pm 0.01 \% \mathrm{IA} / \mathrm{g}$ for adrenal glands; $0.17 \pm 0.09 \mathrm{vs} .0 .03 \pm$ $0.01 \% \mathrm{IA} / \mathrm{g}$ for spleen; $0.14 \pm 0.04$ vs. $0.03 \pm 0.01 \% \mathrm{IA} / \mathrm{g}$ for bone marrow). In addition, significantly higher activities were measured in the right atherosclerotic carotid arteries and abdominal aortas in comparison to the noninjured left carotid arteries and abdominal aortas of control rabbits $(0.08 \pm 0.02 \% \mathrm{IA} / \mathrm{g}$ and $0.10 \pm$ $0.03 \% \mathrm{IA} / \mathrm{g}$ vs. $0.05 \pm 0.01 \% \mathrm{IA} / \mathrm{g}$ and $0.05 \% \pm 0.01 \% \mathrm{IA} / \mathrm{g}$, respectively, $P<0.05$ for both).

\section{Correlation Between ${ }^{125}$ I-Pentixafor Accumulation and Expression of CXCR4 in Plaques}

CXCR4 expression was mostly located in macrophage-rich (RAM-11-positive) regions of atherosclerotic plaques (Fig. 4). The percentage of CXCR4-positive immunostaining was significantly higher in atherosclerotic plaques of the abdominal aorta and right carotid artery relative to the corresponding segments of control rabbits $(75 \% \pm 25 \%$ vs. $26 \% \pm 13 \%$ and $58 \% \pm 15 \%$ vs. $23 \% \pm 16 \% ; P<0.05$ for both; Fig. 5). In addition, atherosclerotic plaque sections that exhibited high-counting rates, as detected by autoradiography, contained high CXCR4 expression in the corresponding adjacent histologic sections (Fig. 4). In contrast, atherosclerotic plaques with low autoradiographic activities as well as normal aortic walls from control rabbits contained only few cells expressing CXCR4. A strong correlation $\left(r^{2}=0.61 ; P<\right.$

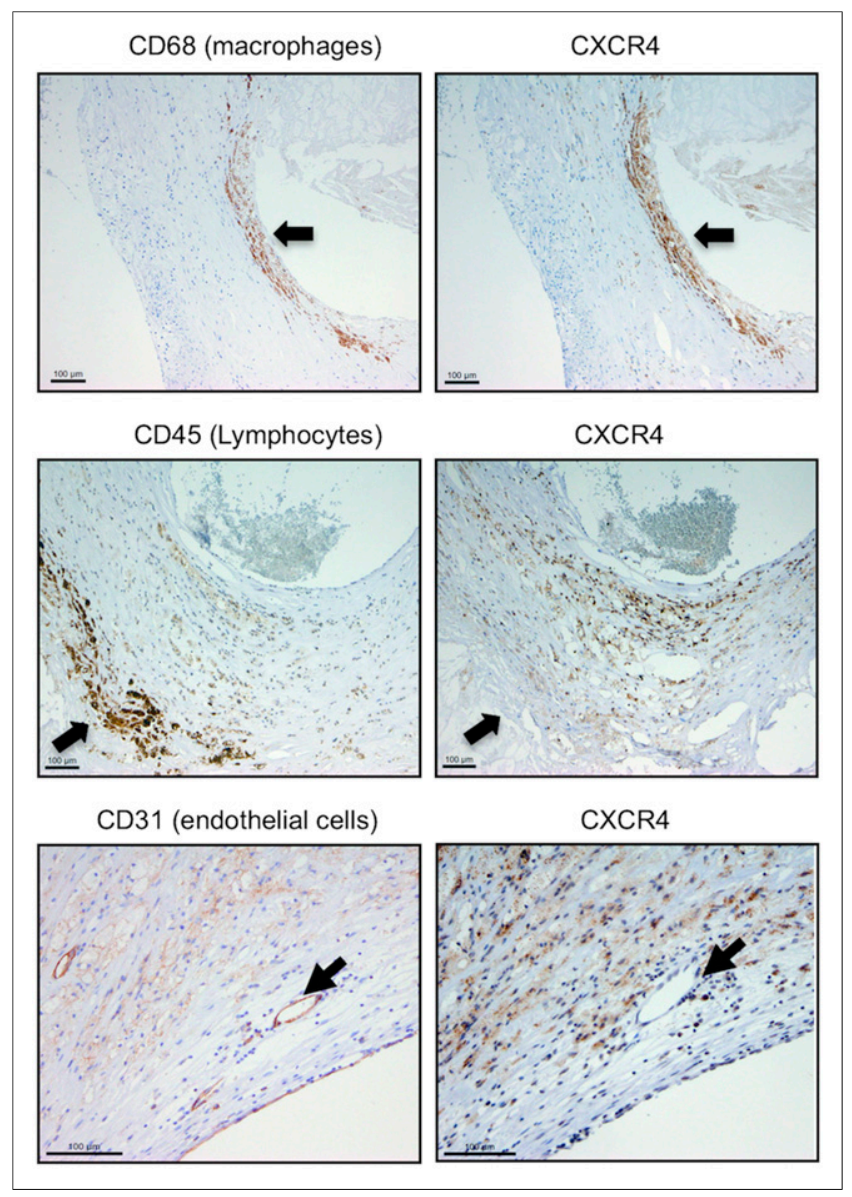

FIGURE 7. Representative examples of CXCR4 expression in human carotid plaques on immunohistochemistry. In human carotid plaques, CXCR4 expression by immunohistochemistry was mostly located in macrophage-rich areas (CD-68-positive cells), whereas only weak expression of CXCR4 was present in lymphocyte-rich areas (CD-45-positive cells) and in endothelial cells (CD-31-positive cells).

0.05) was found between the mean activity quantified on autoradiography in arterial sections and the percentage of CXCR4-positive areas measured in the arterial wall on adjacent histologic sections by immunohistology (Fig. 5). Interestingly, the addition of oxidized lowdensity lipoprotein to macrophage cell culture induced a strong increase in the expression of CXCR4 at the cell surface detected with fluorescence-activated cell sorting analysis (Fig. 5; 17,892 \pm 5,964 vs. $39,157 \pm 22,285 ; P<0.05$ ), whereas incubation with tumor necrosis factor $\alpha$ did not have any effect on CXCR4 expression on macrophages $(17,892 \pm 5,964$ vs. $18,200 \pm 4,683 ; P>0.05)$. Incubation with statins did not have any effect on CXCR4 expression at the surface of macrophages incubated with oxidized low-density lipoprotein $(48,600 \pm 6,520$ vs. $39,157 \pm 22,285 ; P>0.05)$.

\section{Detection of ${ }^{68} \mathrm{Ga}$-Pentixafor Uptake with PET in Human Carotid Plaques}

${ }^{68} \mathrm{Ga}$-pentixafor uptake in carotid arteries was analyzed with PET/MR imaging in 4 patients with carotid stenosis greater than $50 \%$ and 4 patients with carotid stenosis less than $30 \%$. Focal, intense uptake of ${ }^{68} \mathrm{Ga}$-pentixafor $\left(\mathrm{SUV}_{\max }=3.4\right.$ and 2.1 , and most-diseasedsegment TBR $=2.6$ and 2.1) was identified in carotid atherosclerotic plaques of 2 of 4 patients with carotid stenosis greater than $50 \%$ (Supplemental Table 1; supplemental materials are available at 
http://jnm.snmjournals.org). Interestingly, 1 patient had presented an ischemic stroke for $2 \mathrm{wk}$ and showed high uptake of ${ }^{68} \mathrm{Ga}$-pentixafor in the carotid plaques ipsilateral to the territory of the stroke (Fig. 6). Intense accumulation of ${ }^{68} \mathrm{Ga}$-pentixafor could also be detected in perivascular lymph nodes and tonsils. No significant ${ }^{68} \mathrm{Ga}$-pentixafor uptake was detected in the carotid arteries of the patient with asymptomatic carotid stenosis and in patients with carotid stenosis less than $30 \%$. On immunohistochemistry of human carotid plaques (Fig. 7), CXCR4 expression was mostly located in macrophage-rich areas (CD-68-positive cells), whereas only weak expression of CXCR4 was present in lymphocyte-rich areas (CD-45-positive cells) and on endothelial cells (CD-31-positive cells).

\section{DISCUSSION}

In this study, using a rabbit model of atherosclerosis, we demonstrate that the expression of CXCR4 in atherosclerotic plaques can be detected in vivo with PET imaging after injection of ${ }^{68} \mathrm{Ga}-$ pentixafor. A significantly higher uptake of ${ }^{68} \mathrm{Ga}$-pentixafor was detected both in vivo and ex vivo in atherosclerotic plaques of rabbits compared with normal arterial wall and was significantly reduced after a preinjection of the potent CXCR4 inhibitor AMD3100, excluding a nonspecific retention of the tracer in the arterial wall. In addition, the intensity of ${ }^{68} \mathrm{Ga}$-pentixafor uptake in the aortic wall quantified on autoradiography strongly correlated with CXCR4positive areas measured on adjacent histologic sections. Furthermore, we confirmed in a small number of patients that focal ${ }^{68} \mathrm{Ga}$-pentixafor uptake can be detected in carotid atherosclerotic plaques with PET. In human carotid atherosclerotic plaques, we found that CXCR4 expression was localized in macrophage-rich areas. Hence, ${ }^{68} \mathrm{Ga}$-pentixafor represents a promising PET radiotracer for identification of macrophage infiltration present in highrisk atherosclerotic plaques.

\section{Targeting CXCR4 in Atherosclerotic Plaques}

The precise role of CXCR4 expression in atherosclerosis is complex and remains controversial (6). CXCR4 might play a proatherogenic role in attracting leukocytes in plaques but may also have a protective role through the recruitment of endothelial cells. In addition, CXCR4 expression is modulated by heteromerization with CXCR7 (21) or by interaction with hetero-complexes formed of CXCL12 and high-mobility group box 1 proteins (22), underscoring the limitations of messenger RNA and protein expression measurements of CXCR4 performed so far in atherosclerotic plaques. Radiolabeled pentixafor presents the advantage of binding exclusively to CXCR4 expressed on the cell membrane, and its uptake might therefore give a more precise estimation of the location and number of functional CXCR4 proteins in atherosclerotic plaques. Interestingly, we evidenced in this study a strong correlation between the intensity of radiolabeled pentixafor uptake in plaques and the expression of CXCR4 on immunohistochemistry. Radiolabeled pentixafor might thus provide an interesting opportunity to understand more in depth the functional expression of CXCR4 in plaques and its changes over time using in vivo PET imaging.

\section{Imaging Macrophages in Atherosclerotic Plaques with ${ }^{68} \mathrm{Ga}$-Pentixafor}

To be relevant for the evaluation of atherosclerotic plaques, the biologic target of a radiotracer needs to fulfill several conditions: to be sufficiently expressed in plaques so that the radiotracer can reach concentrations allowing for its detection with PET, to differ significantly between high-risk and stable plaques, and to be associated with the risk of subsequent plaque rupture and clinical events. On the basis of these criteria, macrophages appear as a relevant target for atherosclerosis imaging: high macrophage density can be found in atherosclerotic plaques (23), the number of macrophages is significantly higher in complicated versus noncomplicated plaques $(24,25)$, and the identification of macrophages in atherosclerotic plaques has been associated with an increased risk of cardiovascular events during follow-up (26). Interestingly, we found in this study that radiolabeled pentixafor accumulated in macrophage-rich regions of rabbit and human atherosclerotic plaques. By the means of fluorescence-activated cell sorting analysis, we furthermore confirmed that a low-density lipoprotein-rich milieu induced an increase in the expression of CXCR4 at the surface of macrophages. Hence, ${ }^{68} \mathrm{Ga}$-pentixafor appears relevant for the evaluation of atherosclerotic plaques as a noninvasive imaging marker of macrophage infiltration in the vessel wall.

\section{Advantages of ${ }^{68} \mathrm{Ga}$-Pentixafor Over Existing PET Radiotracers in Atherosclerosis Imaging}

Over the past $10 \mathrm{y}$, several PET radiotracers have been developed for the imaging of atherosclerotic plaques, each of them targeting specific biologic activities, cellular types, or protein expression increased in high-risk plaques (27). Nevertheless, ${ }^{68} \mathrm{Ga}$-pentixafor might hold several advantages over existing PET radiotracers in this indication. First, the intensity of the signal in atherosclerotic plaques was high enough to be detectable with PET. Second, physiologic expression of CXCR4 is limited to the spleen, adrenal glands, and bone marrow. ${ }^{68} \mathrm{Ga}$-pentixafor uptake in atherosclerotic plaques can thus be precisely assessed thanks to a low background signal in tissues adjacent to the arterial wall (myocardium, brain, muscle). Third, ${ }^{68} \mathrm{Ga}$-pentixafor-PET imaging does not require the patient to fast as is the case for ${ }^{18} \mathrm{~F}-\mathrm{FDG}$. Fourth, pentixafor can readily be radiolabeled with the generator nuclide ${ }^{68} \mathrm{Ga}$, which is obtained after elution from a ${ }^{68} \mathrm{Ge} /{ }^{68} \mathrm{Ga}$ generator and does not require the use of an on-site cyclotron. The flexibility of this synthesis could be particularly interesting for cardiovascular imaging. Taken together, ${ }^{68} \mathrm{Ga}$-pentixafor might greatly facilitate the noninvasive detection of CXCR4 expression and macrophage infiltration in atherosclerotic plaques with PET.

This study shows minor limitations. First, additional studies are required to unravel in more details the cellular processes in which CXCR4 is involved and the underlying molecular mechanisms associated with its expression in atherosclerotic plaques. In this context, the understanding of the complex role of CXCR4 will be significantly improved by the availability of an imaging method allowing for the in vivo monitoring of CXCR4 receptor expression in atherosclerotic plaques. Of note, radiolabeled pentixafor shows only a low affinity for murine CXCR4 and can therefore not be used to follow CXCR4 expression over time in an atherosclerotic mice model. Second, the level of CXCR4 expression on macrophages measured using fluorescence-activated cell sorting analysis was not significantly affected by ex vivo incubation with statins. Additional experiments are, however, required to test the effects of systemic lipid-lowering on CXCR4 expression at the surface of plaque macrophages. Third, definite conclusions cannot be drawn on the value of ${ }^{68} \mathrm{Ga}$-pentixafor PET imaging for plaque imaging because the number of patients evaluated in this study was small. In a forthcoming study, we plan to evaluate in 45 additional patients with high-grade carotid stenosis the interest of ${ }^{68} \mathrm{Ga}$-pentixafor 
PET imaging for the detection of CXCR4 expression in plaques and to compare the intensities of ${ }^{68} \mathrm{Ga}$-pentixafor and ${ }^{18} \mathrm{~F}$-FDG uptake in human plaques. The results of this clinical study will give us more hints on the value of ${ }^{68} \mathrm{Ga}$-pentixafor PET imaging for the evaluation of atherosclerotic plaques. Finally, whether the intensity of ${ }^{68} \mathrm{Ga}$-pentixafor uptake detected with PET in the vascular wall represents a relevant biomarker to predict the risk of plaque rupture and subsequent clinical events will need to be validated in future studies.

\section{CONCLUSION}

In this study, we demonstrated that the intensity of CXCR4 expression in atherosclerotic plaques could be assessed with ${ }^{68} \mathrm{Ga}$ pentixafor PET imaging in a rabbit model. In addition, we have confirmed in a small number of patients that ${ }^{68} \mathrm{Ga}$-pentixafor uptake can be detected in human carotid plaques. Taken together, these results support a potential role of ${ }^{68} \mathrm{Ga}$-pentixafor PET imaging for the more specific identification of macrophages in atherosclerotic plaques by overcoming the current limitations of ${ }^{18} \mathrm{~F}-\mathrm{FDG}$ for the detection of inflammation in the cardiovascular field. Future studies will need to be performed to understand more in depth the origin, meaning, and changes of CXCR4 expression in tissues taking place during inflammatory processes. In fact, the interest of ${ }^{68} \mathrm{Ga}$-pentixafor PET imaging is not restricted to atherosclerosis imaging but might also find a role for the more specific detection of inflammatory cells in organs with intrinsic high ${ }^{18} \mathrm{~F}-\mathrm{FDG}$ uptake such as the heart.

\section{DISCLOSURE}

This work was supported by the Advanced Research Grant "Multimodal Molecular Imaging" (MUMI; grant 294582; European Research Council Executive Agency), by the Deutsche Forschungsgemeinschaft (DFG; Grossgeräteinitiative and SFB 824; subprojects B5 to Prof. Hans-Jürgen Wester and Z2 to Katja Steiger), by the High-Risk High-Volume grant from the Deutsches Zentrum für Herz-Kreislauf Forschung (DZHK) to Prof. Markus Schwaiger, and by the Deutsche Forschungsgemeinschaft (SFB 1123-A1) to Yvonne Döring and Prof. Christian Weber. Hans-Jürgen Wester is shareholder of SCINTOMICS, Germany. No other potential conflict of interest relevant to this article was reported.

\section{ACKNOWLEDGMENTS}

We thank Sylvia Schachoff, Claudia Meisinger, and Anna Winter for their valuable help in acquiring PET/MR images; Sybille Reder for her valuable support in animal experiments; and Renate Hegenloh for her expertise in histologic analysis of atherosclerotic plaques.

\section{REFERENCES}

1. Rudd JH, Hyafil F, Fayad ZA. Inflammation imaging in atherosclerosis. Arterioscler Thromb Vasc Biol. 2009;29:1009-1016.

2. Hyafil F, Cornily JC, Rudd JH, Machac J, Feldman LJ, Fayad ZA. Quantification of inflammation within rabbit atherosclerotic plaques using the macrophagespecific CT contrast agent N1177: a comparison with ${ }^{18} \mathrm{~F}-\mathrm{FDG}$ PET/CT and histology. J Nucl Med. 2009;50:959-965.
3. Figueroa AL, Abdelbaky A, Truong QA, et al. Measurement of arterial activity on routine FDG PET/CT images improves prediction of risk of future CV events. JACC Cardiovasc Imaging. 2013;6:1250-1259.

4. Bucerius J, Hyafil F, Verberne HJ, et al. Position paper of the cardiovascular Committee of the European Association of Nuclear Medicine (EANM) on PET imaging of atherosclerosis. Eur J Nucl Med Mol Imaging. 2016;43: 780-792.

5. Demmer O, Gourni E, Schumacher U, Kessler H, Wester HJ. PET imaging of CXCR4 receptors in cancer by a new optimized ligand. ChemMedChem. 2011;6:1789-1791.

6. Döring Y, Pawig L, Weber C, Noels H. The CXCL12/CXCR4 chemokine ligand/ receptor axis in cardiovascular disease. Front Physiol. 2014;5:212.

7. Gourni E, Demmer O, Schottelius M, et al. PET of CXCR4 expression by a ${ }^{68} \mathrm{Ga}-$ labeled highly specific targeted contrast agent. J Nucl Med. 2011;52:1803-1810.

8. Wester HJ, Keller U, Schottelius M, et al. Disclosing the CXCR4 expression in lymphoproliferative diseases by targeted molecular imaging. Theranostics. 2015; 5:618-630.

9. Philipp-Abbrederis K, Herrmann K, Knop S, et al. In vivo molecular imaging of chemokine receptor CXCR4 expression in patients with advanced multiple myeloma. EMBO Mol Med. 2015;7:477-487.

10. Herrmann K, Lapa C, Wester HJ, et al. Biodistribution and radiation dosimetry for the chemokine receptor CXCR4-targeting probe ${ }^{68} \mathrm{Ga}$-pentixafor. $\mathrm{J} \mathrm{Nucl}$ Med. 2015;56:410-416.

11. Debnath B, Xu S, Grande F, Garofalo A, Neamati N. Small molecule inhibitors of CXCR4. Theranostics. 2013;3:47-75.

12. Schioppa T, Uranchimeg B, Saccani A, et al. Regulation of the chemokine receptor CXCR4 by hypoxia. J Exp Med. 2003;198:1391-1402.

13. Martin R, Juttler S, Muller M, Wester HJ. Cationic eluate pretreatment for automated synthesis of $\left[{ }^{68} \mathrm{Ga}\right]$ CPCR4.2. Nucl Med Biol. 2014;41:84-89.

14. Mueller D, Klette I, Baum RP, Gottschaldt M, Schultz MK, Breeman WA. Simplified $\mathrm{NaCl}$ based ${ }^{68} \mathrm{Ga}$ concentration and labeling procedure for rapid synthesis of ${ }^{68} \mathrm{Ga}$ radiopharmaceuticals in high radiochemical purity. Bioconjug Chem. 2012;23:1712-1717.

15. Hyafil F, Cornily J, Feig J, et al. Noninvasive detection of macrophages using a nanoparticulate contrast agent for computed tomography. Nat Med. 2007;13: 636-641.

16. Hutton BF, Braun M, Thurfjell L, Lau DY. Image registration: an essential tool for nuclear medicine. Eur J Nucl Med Mol Imaging. 2002;29:559-577.

17. Rosset A, Spadola L, Ratib O. Osirix: an open-source software for navigating in multidimensional Dicom images. J Digit Imaging. 2004;17:205-216.

18. Rudd JH, Myers KS, Bansilal S, et al. Atherosclerosis inflammation imaging with ${ }^{18}$ F-FDG PET: carotid, iliac, and femoral uptake reproducibility, quantification methods, and recommendations. J Nucl Med. 2008;49:871-878.

19. Martinez-Moller A, Souvatzoglou M, Delso G, et al. Tissue classification as a potential approach for attenuation correction in whole-body PET/MRI: evaluation with PET/CT data. J Nucl Med. 2009;50:520-526.

20. Delso G, Furst S, Jakoby B, et al. Performance measurements of the Siemens mMR integrated whole-body PET/MR scanner. J Nucl Med. 2011;52:19141922.

21. Decaillot FM, Kazmi MA, Lin Y, Ray-Saha S, Sakmar TP, Sachdev P. CXCR7/ CXCR4 heterodimer constitutively recruits Beta-arrestin to enhance cell migration. J Biol Chem. 2011;286:32188-32197.

22. Schiraldi M, Raucci A, Munoz LM, et al. HMGB1 promotes recruitment of inflammatory cells to damaged tissues by forming a complex with CXCL12 and signaling via CXCR4. J Exp Med. 2012;209:551-563.

23. Kolodgie FD, Narula J, Burke AP, et al. Localization of apoptotic macrophages at the site of plaque rupture in sudden coronary death. Am J Pathol. 2000; 157:1259-1268.

24. Narula J, Nakano M, Virmani R, et al. Histopathologic characteristics of atherosclerotic coronary disease and implications of the findings for the invasive and noninvasive detection of vulnerable plaques. J Am Coll Cardiol. 2013;61:10411051.

25. Figueroa AL, Subramanian SS, Cury RC, et al. Distribution of inflammation within carotid atherosclerotic plaques with high-risk morphological features: a comparison between positron emission tomography activity, plaque morphology, and histopathology. Circ Cardiovasc Imaging. 2012;5:69-77.

26. Marnane M, Prendeville S, McDonnell C, et al. Plaque inflammation and unstable morphology are associated with early stroke recurrence in symptomatic carotid stenosis. Stroke. 2014;45:801-806.

27. Tarkin JM, Dweck MR, Evans NR, et al. Imaging atherosclerosis. Circ Res. 2016;118:750-769. 